\title{
Théologiques
}

\section{La vie dans les contes et fables d'Afrique noire}

\section{Ntumba Muena Muanza}

Volume 19, numéro 1, 2011

Théologie africaine et vie

URI : https://id.erudit.org/iderudit/1014180ar

DOI : https://doi.org/10.7202/1014180ar

Aller au sommaire du numéro

Éditeur(s)

Faculté de théologie et de sciences des religions, Université de Montréal

ISSN

1188-7109 (imprimé)

1492-1413 (numérique)

Découvrir la revue

Citer cet article

Muena Muanza, N. (2011). La vie dans les contes et fables d'Afrique noire. Théologiques, 19(1), 47-69. https://doi.org/10.7202/1014180ar

\section{Résumé de l'article}

Oeuvre de liturgie et d'anthropologie esthétique, cette publication révèle la vitalité d'une communauté chrétienne d'Afrique centrale (du Congo Kinshasa, à Cikapa) qui affronte ses problèmes de survie, dans une liturgie eucharistique dominicale, émaillée de contes comme quatrième lecture. Ces contes y apparaissent comme un moment important de Révélation en Afrique et un moyen mnémotechnique puissant d'éducation et d'action populaire. Y est interprétée, de manière critique et métaphorique, la vie des sociétés postcoloniales, dominées par un capitalisme commercial sauvage avec ses méthodes immorales d'accumulation, au détriment de la majorité et au service d'une minorité interne liée à l'impérialisme. La vie apparaît aussi dans la créativité artistique même des contes qui ne sont jamais figés et dans la manière vivante de les déclamer. Les contes sont une constante réinterprétation de la vie dans le miroir culturel africain et chrétien.
Ce document est protégé par la loi sur le droit d'auteur. L'utilisation des services d'Érudit (y compris la reproduction) est assujettie à sa politique d'utilisation que vous pouvez consulter en ligne.

https://apropos.erudit.org/fr/usagers/politique-dutilisation/ 


\title{
La vie dans les contes et fables d'Afrique noire
}

\author{
Ntumba Muena Muanza \\ Anthropologie \\ Institut Supérieur Pédagogique, Cikapa (RDC)
}

On a souvent affirmé que les civilisations de l'oralité, dont celles de l'Afrique noire, ne sont pas celles de la pensée logique; ce sur quoi renchérit un célèbre écrivain et homme politique africain: "la raison est hellène et l'émotion est nègre» (L. S. Senghor); ainsi les Noirs ne rayonneraient que quand ils chantent et dansent, rejoignant ainsi la célèbre phrase de Mobutu: «heureux le peuple qui chante et qui danse ». Images figées, idées fausses communément propagées et admises. De véritables mensonges qui s'incrustent dans les têtes de plusieurs, forgeant une fausse conscience que les Noirs ont d'eux-mêmes.

Les fables, les contes, les proverbes, les chansons, réservoir inépuisable de l'anthropologie esthétique, sont ainsi souvent conçus comme des moments de loisir, de simple amusement. Et pourtant, ces divers codes esthétiques de communication sociale, pleins de métaphores, donnent à penser. Contes et fables sont des récits fictifs, inventés et montés exprès pour faire comprendre la vie réelle.

Ils sont racontés de manière vivante, chantés au clair de lune, mais aussi à diverses occasions qui émaillent les moments de la vie individuelle et collective en Afrique noire précoloniale. Depuis la domination de l'Afrique noire par le capitalisme triomphant, les idées souvent véhiculées par les écoles et les religions importées ont combattu contes et proverbes, chants et danses, considérés comme sphères d'action satanique (bintu bya beena Dyabolo $)^{1}$.

Ces attitudes négatives à l'égard des contes, attitudes "culturocides", n’ont pas fondamentalement changé, 51 ans après l'indépendance. Dans

1. L'épisode de l'acharnement d'un prêtre noir pour casser un tambour traditionnel en dit long (voir Kabasele Lumbala et al. 2011, 57-58). 
plusieurs églises néocoloniales d'Afrique noire, les contes, les mythes, les mélodies... africains n'ont pas droit de cité. Depuis l'éclosion des discours de la conscience noire avec Césaire, Senghor, Cheikh Anta Diop et dans la foulée des luttes anticoloniales de Fanon, qui ont culminé dans le combat de libération religieuse et de l'inculturation (de Mgr Tshibangu, du cardinal Malula, de Bimwenyi, de Mveng, etc.), un autre monde apparaît à l'horizon. C'est dans cette optique qu'une expérience osée de bouleversement total des discours et pratiques d'aliénation est tentée dans une paroisse de la ville de Cikapa en République démocratique du Congo, Paroisse Budikadidi St Joseph, au diocèse de Luebo, depuis 1985, avec, entre autres, l'introduction d'une déclamation d'un conte traditionnel durant la célébration dominicale. Dans le rituel africain de l'eucharistie, le conte est considéré comme un lieu important de révélation de Dieu aux Africains à travers sa Parole, une parole vivifiante visant essentiellement le triomphe de la vie sur la mort. Les contes visent soit à renforcer le peuple noir dans sa vie autour de certaines valeurs, soit à l'aguerrir contre les pièges divers tendus dans la vie individuelle et collective.

Ces contes traditionnels ne sont pas simplement repris, déclamés comme dans la tradition; ils sont également recréés à Budikadidi, depuis 1986-1987, suivant des thèmes bien précis qui traitent de la vie africaine actuelle, analysée en assemblée paroissiale ${ }^{2}$ de septembre ou d'octobre. Ils constituent, selon les choix auxquels nous nous sommes astreints, la quatrième lecture chaque dimanche, parce qu'ils sont un repère important de la Révélation en Afrique et un moyen mnémotechnique d'envergure. C'est grâce à cette expérience que nous nous sommes familiarisés avec les fables et contes africains, et que nous en avons rassemblé jusqu'à présent plus de 10000 , dont un petit aperçu a été édité dans une publication, il y a quelques années (Muena Muanza et Kabasele Lumbala 2001).

Les contes et fables racontent donc et interprètent nécessairement la vie africaine dans le contexte postcolonial. La vie des Africains y est décrite sous plusieurs facettes. Les fables sont des récits dont les personnages sont principalement les animaux, tandis que les personnages du conte sont divers; mais ce n'est pas ici le lieu de nous étendre sur les caractéristiques

2. L'assemblée paroissiale qui regroupe les divers responsables chrétiens, élus dans des communautés de base et dans les mouvements d'action catholique, est conçue comme l'organe suprême de décision de la paroisse, en dehors des structures de dialogue du Concile Vatican II. 
des contes, telles que versification et autres; nous nous arrêtons à l'expression de la vie dans nos fables et contes.

Dans un premier temps, je mentionnerai un échantillon de contes et fables; puis je tenterai, dans un second temps, de montrer comment la vie africaine s'y déploie et s'y exprime.

\section{Quelques contes et fables d'Afrique noire racontés pendant la célébration eucharistique à la paroisse Budikadidi (Cikapa-Kele) ${ }^{3}$}

\subsection{Le crapaud, grand escroc}

Le crapaud voulait organiser une grande fête à l'occasion de la première communion de son fils (ou de sa sortie de l'initiation); mais il n'en avait pas les moyens. Exploitant les faiblesses de chacun des animaux suivants (cancrelat, poule, boa, léopard, éléphant), il décida de leur emprunter ce dont il avait besoin pour fêter. Il emprunta: 4 seaux de maïs de la part du cancrelat, 4 seaux de manioc de la poule, 10 calebasses de vin de palme du boa, une grosse chèvre du léopard, un porc et 200 dollars de l'éléphant. Il promit de remettre à chacun de ses créanciers son dû dès le lendemain de la fête; il prit rendez-vous avec chacun à une heure précise, dès 7 heures du matin.

Sa femme s'exclama, réagissant fortement: «tu n'as pas de moyens, comment vas-tu remettre tous ces biens? Je risque de me retrouver empêtrée dans des combines où mes nerfs craqueront; aussi je te quitte et retourne chez mes parents! " La réponse du crapaud fut rapide: "ne pars pas, je remettrai tout sans histoires, tu verras ».

La fête fut bien organisée, à la grande satisfaction de tous. Les gens mangèrent du bidya, du riz, des ignames, des bananes plantains frites dans l'huile de palme, différentes viandes (chèvre, porc, gibier, volailles, poissons). L'eau fraîche et les vins de palme coulaient à flots. Le lendemain de la fête, le cancrelat vint dès 7 heures du matin récupérer son bassin de maïs. Le crapaud l'accueillit avec style, et lui offrit de l'alcool, dont il avait acheté à dessein cinq bouteilles. Le cancrelat en but et s'enivra. Le crapaud le plaça sur un lit. La poule vint à son tour dès 8 heures pour réclamer son bassin de manioc. Le crapaud le reçut avec gentillesse et lui dit: je viens de causer avec le cancrelat tout à l'heure. Et la poule de réagir: où est le

3. Je rends ici hommage aux grands conteurs de la paroisse Budikadidi qui se sont éteints: Kayoko Mpumbu, Wela Dibungi, Cyela Bwadi, Malumba Citadi, Mundi wa Cikela, Mputu Cinwa mayi, Ngalamulume Dibunda... 
cancrelat? Le crapaud lui montra où il dormait. La poule le picota et l'avala en une seconde. Le crapaud, après avoir réglé le cas du cancrelat, invita la poule à boire un peu d'alcool avant de lui remettre son bassin de manioc. La poule prit du plaisir à boire et s'enivra; on la déposa sur le lit. Mukenge s'amena à son tour dès 9 heures chez le crapaud. Mukenge pourchasse naturellement les poules comme son mets préféré. Le crapaud l'accueillit avec joie et lui annonça une bonne nouvelle: la présence de la poule chez lui. Mukenge, étonné, lui demanda: où est la poule? Viens voir, lui répondit le crapaud. Mukenge se jeta sur la poule, la disséqua et la mangea délicieusement. Après son repas préféré, il fut invité à boire par le crapaud. Il but énormément et s'enivra à son tour. On le mit sur le célèbre lit d'après le rituel.

Le boa vint lui aussi réclamer son vin de palme dès 10 heures, lui qui aime avaler les bêtes moyennes. Le crapaud l'accueillit très bien en lui annonçant une très bonne nouvelle, celle de la présence de la petite bête Mukenge chez lui; le boa jubila et se jeta sur la petite bête et l'avala. Le crapaud lui apporta de l'alcool près du lit où il s'était allongé, incapable de bouger. Il but et s'enivra lui aussi. Même scénario à l'arrivée du léopard pour réclamer sa chèvre. Il se régala avec la viande de boa et assouvit sa soif avec l'alcool. Il but et s'enivra à son tour. Il fut placé lui aussi sur le même lit où l'attendait sa mort, qui survint à la venue de l'éléphant pour la réclamation de son porc ainsi que de ses 200 dollars dès 11 heures. Comme l'éléphant était à la recherche d'une peau de léopard, il profita de l'occasion qui lui était offerte pour l'avoir et manger la viande du léopard. Et en compensation de ses 200 dollars, il prit la peau du léopard. C'est ainsi que le crapaud avait fini par régler ses comptes avec tous les animaux qui lui avaient prêté leurs biens, mais à leurs dépens.

\subsection{Citeya, attrapeur de rats}

Le métier secondaire de Citeya, à part l'agriculture, consistait à tendre des pièges aux rats. Il en était devenu expert et en attrapait beaucoup en les enfilant dans sa gibecière. Il devait cependant lui aussi affronter un piège important chaque fois à son retour de son activité: les femmes qui se lavaient presque nues au ruisseau. Il devait passer obligatoirement par ce ruisseau pour rentrer à la maison.

En arrivant près du ruisseau, les femmes s'approchaient de lui en lui exposant leurs seins et leurs ventres, embellis par l'esthétique africaine des tatouages. Elles lui disaient, en le piégeant: «notre chéri Citeya, regarde et 
donne-nous des rats ». Hypnotisé, Citeya leur remettait les rats sans compter et il s'étonnait de ne plus rien avoir dans sa gibecière à la fin. Il repartait ainsi bredouille à la maison, laissant sa femme et ses enfants affamés. Il les exposait ainsi à la mort.

Ainsi, tendeur de pièges, il tombait souvent lui-même dans le piège que lui tendaient les femmes au ruisseau. Cela arriva plusieurs fois, au détriment de sa famille. Un jour, la force surnaturelle alliée à la vie (cikulu-cikaji) lui apparut et l'avertit. Elle lui dit: "essaie de t'en sortir en tentant de toucher les seins de ces femmes; tu verras ce qu'elles vont faire parce qu'elles suivent scrupuleusement leur coutume qui interdit de s'amuser avec des hommes autres que leur mari ". Citeya suivit les consignes en arrivant auprès des femmes au ruisseau. Elles lui montrèrent leurs seins et leurs ventres, Citeya saisit l'occasion, et tendit sa main pour caresser le sein d'une des femmes; les femmes s'enfuirent et s'en allèrent loin de Citeya. C'est ainsi qu'il a pu échapper au piège, et repartir avec tous ses rats, au grand bonheur de sa famille et de tous les siens.

\subsection{Le caméléon et le coq}

Le caméléon organisa une grande fête, où il avait invité plusieurs animaux, dont le coq. Tout le monde avait pris place dans la salle des convives. Le caméléon, sa femme et ses enfants se mirent à servir les invités. En apportant verres et boissons, ils changèrent de couleur, en prenant la couleur des verres et de la boisson. En servant le repas, ils changeaient de couleur en prenant celle des assiettes. Puis en servant les feuilles de manioc, ils prirent la couleur verte, et successivement, la couleur de la viande, puis celle rougeâtre des fruits pour le dessert. Tout était bien assorti.

Les invités mangèrent et burent à leur faim. Mais le coq était tellement impressionné par ce spectacle, qu'il brûla lui aussi d'envie d'organiser une séance semblable dans un festin où il changerait successivement de couleur selon les plats et les objets qu'il manipulerait, à l'exemple du caméléon. Il invita de nombreux animaux, et s'informa auprès du caméléon de sa technique pour changer de couleur; le caméléon lui dit qu'il ne faisait absolument rien de spécial, et qu'il lui suffisait de s'approcher d'un récipient pour que la couleur de sa peau s'harmonise avec le récipient. Le coq essaya, mais ne réussit pas; il pensa que les récipients du caméléon étaient spéciaux et les emprunta; mais les effets ne suivaient pas; les invitations ayant déjà été faites, le coq attendit le jour avec angoisse, car il ne voulait pas annuler la fête. 
Ayant placé les invités aux différentes places prévues, le coq entra dans sa chambre et demanda à sa femme de le changer de forme en enlevant les plumes des ailerons. Sous cette forme, il empoigna les verres et la boisson, et se mit à servir. Puis, disparaissant de nouveau dans sa chambre, il appela sa femme et lui demanda de le changer en enlevant les plumes de sa queue; il sortit alors, muni des assiettes et du repas principal. Vers la fin du repas, il disparut de nouveau pour s'enfermer dans sa chambre, en compagnie toujours de sa femme, et lui demanda de le déplumer complètement; sous cette dernière forme, il ramena le dessert aux invités qui étaient à chaque fois éberlués, tout autant que satisfaits de la qualité du repas.

Après avoir congédié les invités, le coq demeura dans sa chambre, tant il avait pris froid. Dehors, une pluie diluvienne s'abattit, pour empirer la situation déjà critique. On alluma un feu dans la maison, et le coq s'en approcha; mais il avait toujours l'impression que le feu ne suffisait pas; il fit augmenter la quantité de bois, dans l'espoir que le feu le réchaufferait; mais au fur et à mesure que le feu augmentait, son corps rougissait et des démangeaisons terribles le prirent sur tout le corps; il se mit à enfler, et mourut peu après des suites des brûlures.

\subsection{Le léopard, le lion et le pangolin, et les trois tam-tams 4}

Il y avait une fois, dans une contrée, deux tam-tams qui se relayaient, matin et soir, au lever et au coucher du soleil; le premier tambourinait ce refrain: "Dans cette contrée, nous sommes deux; Seigneur Léopard à la peau reluisante, qui règne sur les forêts, et Sa majesté Lion, à l'allure foudroyante, qui trône dans la savane. » Puis, à l'autre bout, un autre tam-tam reprenait: "oui, dans cette contrée il n'y a que deux voix; celle du Seigneur Léopard à la peau reluisante, et celle de Sa majesté Lion à l'allure foudroyante». Pendant que leurs tambourineurs égrenaient leurs louanges, le léopard se léchait les babines tandis que le lion secouait fièrement sa crinière touffue.

Le pangolin, animal des eaux, fut irrité par cette déclaration qui subjuguait tous les animaux au pouvoir du lion et du léopard. Il alla s'entretenir avec des animaux qui vivaient dans l'eau, et successivement avec l'hippopotame, le crocodile, les grosses anguilles et les poissons. Il leur parla d'un projet de résistance pour affirmer aussi le règne du monde des eaux; car pour lui, le monde des eaux, même si les eaux existent dans la

4. J'emprunte ici la traduction française de ce conte à Kabasele Lumbala (1997). 
savane et dans la forêt, constitue un règne à part entière. Mais hélas, ni l'hippopotame, ni le crocodile, ni les anguilles, ni aucun autre animal des eaux n'avait le courage d'entreprendre une telle démarche.

Aussi le pangolin se décida-t-il de commencer par faire une brèche. Il endossa ainsi le projet de résistance et de révolte du règne animal des rivières et fit alors un tam-tam pour clamer un contre-message. Après le retentissement des tam-tams des deux promontoires qui se voulaient uniques, le troisième tam-tam du pangolin se mit à sonner. Dès que les deux tam-tams du lion et du léopard avaient terminé leur refrain quotidien: "Dans cette contrée, nous sommes deux; Seigneur Léopard à la peau reluisante, qui règne sur les forêts, et Sa majesté Lion, à l'allure foudroyante, qui trône dans la savane ", un troisième tam-tam résonna du fond d'un cours d'eau dans une galerie forestière; et ce troisième tam-tam disait: «Dans cette contrée, nous sommes trois, Seigneur Léopard à la peau reluisante, Sa majesté Lion à l'allure foudroyante, et Maître Pangolin aux écailles tranchantes, qui règne sur les eaux.»

"Comment? répliqua violemment le léopard. Qui est-ce qui prétend ainsi s'ajouter à nous ? cria le lion. Léopard, n'entends-tu pas? Ce tam-tam nouveau semble venir du fond de tes forêts; ne feras-tu rien pour faire taire cet importun?» Le léopard ordonna à son tambourineur de répéter le refrain habituel: "Dans cette contrée, nous sommes deux; Seigneur Léopard à la peau reluisante, qui règne sur les forêts, et Sa majesté Lion, à l'allure foudroyante, qui trône dans la savane »; il fut aussitôt relayé par le tam-tam du lion comme à l'accoutumée; et aussitôt après, le troisième tam-tam résonna de nouveau du fond du cours d'eau en disant: «Dans cette contrée, nous sommes trois, Seigneur Léopard à la peau reluisante, Sa majesté Lion à l'allure foudroyante, et Maître Pangolin aux écailles tranchantes, qui règne sur les eaux. » Pendant ce temps le léopard qui s'était furtivement glissé jusqu'au bord de la rivière, aperçut le petit pangolin avec son tam-tam, perché sur un tronc d'arbre qui flottait sur l'eau. Le léopard fit un bond gigantesque, fonçant sur le pangolin; mais celui-ci, très agile dans l'eau, s'était retourné avec le tronc d'arbre, disparaissant sous l'eau. Le léopard ne trouva sous ses griffes que des copeaux de l'écorce d'arbre, et il se débattit pour ne pas périr dans l'eau; le pangolin en profita même pour le griffer, et le léopard revint bredouille sur la berge. Il comprit qu'en dehors de la savane et de la forêt, il y avait un terrain que ni lui, ni le lion, ne maîtrisaient.

Le soir venu, à l'heure habituelle du refrain des louanges aux deux maîtres, le léopard et le lion écoutèrent en silence les louanges s'égrener, et le troisième tam-tam ajouter la louange du pangolin. 


\subsection{Le tambour des insectes Tukwà-Matèmbwà ${ }^{5}$}

Au village des insectes Tukwà-Matèmbwà, la fabrication des tambours était la principale occupation des habitants. Ces tambours, fabriqués avec un savoir-faire hors pair, étaient joliment décorés de perles et de cuir. Aucun instrument ne soutenait la comparaison. La moindre percussion produisait un son qui incitait irrésistiblement à la danse. Ces tambours étaient tout le temps gardés par de vaillantes sentinelles.

À cette époque, l'éléphant Nzevu était le roi de tous les animaux, fort aimé de son peuple pour sa sollicitude. Aussi était-il l'objet d'un culte grandiose où tambours et xylophones occupaient une place de choix. Un jour, le Roi Nzevu apprit l'existence des tambours des insectes TukwàMatèmbwà grâce à un petit oiseau voyageur. Depuis, habité par la convoitise, le roi décida d'en obtenir par tous les moyens. Il fit venir tous les animaux à la cour, au son du tam-tam. Ils accoururent tous. Personne ne manqua à l'appel. Du haut de son trône, il leur tint ces propos: "Toute personne qui parviendra à m'obtenir un lot de tambours des insectes Tukwà-Matèmbwà, recevra une grande récompense. Un fief lui est réservé; il en sera le seigneur. Toutefois, s'empressa-t-il de les mettre en garde, le chemin qui mène aux tambours des insectes Tukwà-Matèmbwà est périlleux».

Malgré cela, l'empressement fut grand. Chaque animal voulut partir le premier, surtout les grands fauves. Ils finirent par se mettre d'accord et c'est Ntambwa-le-Lion qui fut désigné pour tenter, le premier, sa chance. Avec sa fière allure, Ntambwa invita ses camarades à déjà préparer la fête, son exploit ne faisant aucun doute. Il se mit alors en route vers le village des insectes Tukwà-Matèmbwà. Après avoir très longuement cheminé, il vit soudain le village des insectes Tukwà-Matèmbwà qui, comme d'habitude, étaient occupés à façonner les tambours dans la forêt environnante. La réserve était gardée par des sentinelles. Ayant effectué une reconnaissance des lieux, Ntambwa s'introduisit dans le jardin et, profitant d'un moment de distraction des vigiles, il se glissa dans la réserve. Impressionné par l'éclat et la qualité des tambours, il soupira d'étonnement: "Le Roi Nzevu a bien raison de vouloir à tout prix posséder ces tambours. C'est du jamais vu!»

Mais voilà ! Plutôt que de subtiliser silencieusement les tambours, Ntambwa-le-Lion ne put résister à l'envie de les tester et se laissa aller...

5. J'emprunte la traduction française de ce conte à l'Association culturelle Sangalayi (1996). 
Cette maladresse mit les vigiles en branle. Alors que le lion s'était copieusement servi et s'apprêtait à franchir le seuil de la porte, l'une des sentinelles lança un chant d'alarme pour alerter les autres habitants qui étaient dans la forêt:

Insectes Tukwà-Matèmbwà!

Insectes Tukwà-Matèmbwà!

Njinjinji, ô camarades, njiii!

Nos tambours sont emportés,

Njinjinji, ô camarades, njiii!

Par le peuple des animaux,

Njinjinji, ô camarades, njiii !

Peuple à dents longues,

Njinjinji, ô camarades, njiii!

Peuple à longues oreilles,

Njinjinji, ô camarades, njiii !

Pour les offrir à Nzevu-l'Éléphant !

Njinjinji, ô camarades, njiii!

À cet appel angoissé, les insectes Tukwà-Matèmbwà arrêtèrent de travailler et se précipitèrent au village pour saisir leurs armes. En un clin d'œil, ils encerclèrent Ntambwa et le criblèrent de piqûres. Aucune région du corps de notre fauve n'échappa à la hargne des petites bêtes: même l'intérieur des oreilles ne fut pas épargné. Dépassé par ce qui lui arrivait, Ntambwa se débarrassa des tambours et détala à toutes jambes. Ses comparses, qui l'attendaient, le virent soudain arriver, tout endolori et qui leur disait: «Chers camarades, j’y ai été, ce n'est pas du tout évident!»

C'est alors que Nkashama-le-Léopard, fauve aux ongles terrifiants, se dit prêt à relever le défi. Il ne fut pas long et revint, lui aussi bredouille et surtout, bien amoché. Tous les grands animaux tentèrent l'aventure sans succès. Sa Majesté Nzevu en fut désabusée. C'est alors qu'intervint Nkuvula-Tortue: «Sire, acceptez que je tente ma chance, moi aussi! » Des quolibets fusèrent de la foule: «Que pourras-tu obtenir, traînard que tu es ? Les plus forts d'entre nous y ont été et n'ont rien ramené!" Attentif à toutes ces réactions, Nzevu accorda néanmoins le feu vert à Nkuvu. La tortue se mit en route lentement comme d'habitude, sa carapace lui pesant sur le dos. Une nuit, puis une autre, le chemin était bien long pour elle. Un mois, puis un autre, elle avançait toujours.

La voilà enfin au village des insectes Tukwà-Matèmbwà. Un coup d'œil à gauche, un autre à droite, elle repéra la réserve où étaient stockés les tambours. Les sentinelles ne dormaient même plus, car il fallait protéger 
ces objets précieux que tout le monde vénérait. La tortue savait que les tambours étaient soigneusement gardés. Elle se glissa sous la porte et les vit. Elle en prit deux, les mit sur son dos et les attacha avec une sangle bien solide. Dès que la tortue eut passé la porte, une sentinelle l'aperçut et elle alerta les insectes Tukwà-Matèmbwà avec le chant et le tambour.

Les insectes Tukwà-Matèmbwà accoururent et encerclèrent la tortue. Celle-ci subit un passage à tabac en bonne et due forme: un véritable déluge d'aiguillons. Ayant triomphé des plus gros calibres, les insectes Tukwà-Matèmbwà étaient convaincus qu'ils ne feraient qu'une bouchée de l'indésirable tortue. Mais c'était sans compter avec la résistance de cette dernière dont la carapace en avait vu d'autres. Nkuvu-la-Tortue se laissa faire, mais tout en protégeant la tête sous sa carapace. Les insectes TukwàMatèmbwà, sans désemparer, s'acharnèrent sur l'intruse, mais en vain: leurs aiguillons se cassèrent les uns après les autres sur la carapace de la tortue.

Épuisés, beaucoup d'entre eux s'écroulèrent, sans souffle! Ils laissèrent finalement filer Nkuvu-la-Tortue avec deux tambours. Voyant qu'elle l'avait emporté et que les insectes Tukwà-Matèmbwà étaient retournés dans la forêt, Nkuvu-la-Tortue releva la tête en se disant: «Voici mon jour de gloire, je peux intégrer les rangs des seigneurs. Je confondrai mes moqueurs. »

Ayant longtemps attendu, certains animaux se disaient: "Nous perdons inutilement le temps à attendre Nkuvu-la-Tortue. N'aurait-elle pas déjà crevé sous les coups des piqûres? Il est temps de disposer». Nzevul'Éléphant leur dit: «nous avons déjà tant attendu; patientons encore un peu!» À peine eut-il parlé qu'on vit les tambours briller au loin sur la carapace de la tortue. Forte clameur dans l'assistance. Nzevu-l'Éléphant était aux anges! Nkuvu-la-tortue fut portée en triomphe, et tous ses détracteurs en furent confus! [...] Comme promis, Nkuvu reçut un morceau de territoire, dont les habitants devinrent ses sujets. Elle acquit également tous les insignes du pouvoir: une couronne, une herminette, et une toque de perles et cauris.

\subsection{Les crapauds et les éperviers}

Il existait deux règnes différents: celui des crapauds et celui des éperviers. Les éperviers voulurent régner sur les crapauds en comptant sur leur propre force et leur rapidité, et méprisant les crapauds pour leur lenteur dans la démarche. Le chef des éperviers conçut l'idée d'aller arracher aux 
crapauds leur pouvoir dans la capitale Kinshasa, le siège des institutions politiques et économiques.

En se préparant, l'épervier ouvrit sa mallette pour y placer différents papiers. Sans soupçonner la présence d'une autre personne, l'épervier alla se laver, laissant son sac de voyage ouvert. Le crapaud qui épiait le moment dans un coin de la maison s'introduisit dans son sac de voyage, et s'installa dans un coin. Le moment venu, l'épervier referma le sac, et s'envola vers la capitale. Arrivé dans la soirée, il s'arrangea et prit le rendez-vous pour voir le ministre vers 9 heures le lendemain. Le lendemain matin, ouvrant son sac de voyage, il prit ses articles de toilette, et alla se laver. Aussitôt le crapaud sortit et se précipita chez le ministre où il arriva vers 7 heures du matin.

L'ouverture des bureaux avait lieu à 7 h 30 . Et aussitôt le crapaud entra chez le ministre et présenta ses papiers qui lui octroyaient le pouvoir sur la contrée. Le ministre les ayant signés aussitôt, il obtint ainsi la lettre de confirmation. Puis il regagna les pénates où se trouvait l'épervier. Et le crapaud de l'attendre patiemment dans un coin comme à l'accoutumée. Lorsque l'épervier se présenta plus tard chez le ministre, les secrétaires lui signifièrent que la lettre de confirmation avait déjà été établie pour le crapaud. Malgré ses tentatives de corruption, le ministre refusa de lui octroyer le document qu'il demandait parce que son récipiendaire l'avait déjà reçu une heure auparavant.

L'épervier rentra bredouille, pressé de rentrer à Cikapa pour tromper l'opinion et arracher malgré tout le pouvoir des crapauds. Le crapaud qui était dans le sac de voyage de l'épervier sortit comme à l'accoutumée la nuit; il quitta furtivement la maison de l'épervier et rentra chez lui pour annoncer nuitamment sa prouesse. Dès 5 heures du matin, des cris de liesse éclatèrent partout dans la région, de la part des crapauds, proclamant leur victoire en exhibant la lettre de confirmation du ministre.

\subsection{Les trois fils}

Un père de famille avait trois fils. Il les avait bien éduqués en leur léguant sa richesse: les champs qu'il cultivait, bien situés, dans des terrains fertiles et près des cours d'eau. Mais les fils n'en étaient pas très fiers, car de nouvelles richesses étaient apparues dans le pays, en dehors des champs et de l'agriculture. Ils quittèrent leur village pour rejoindre un autre village où se ramassait du diamant en grande quantité.

En cours de route, un chef qui connaissait leur père jadis, les reconnut et les fit venir chez lui pour leur procurer un peu de repos et des denrées 
pour la route. Se souvenant de la bonté de leur père, de l'amitié qu'ils avaient nouée, et apprenant qu'ils étaient à la poursuite des moyens d'amélioration de leur vie, il leur offrit un colis où il avait mis une liasse de billets de 100 dollars, ainsi qu'un gros diamant de luxe de 100 carats. Ils dirent merci et continuèrent la route.

Ayant fait un bout de chemin, une créature spirituelle alliée à la vie leur apparut et les mit en garde contre ce colis qu'ils venaient de recevoir en disant: "Attention, soyez très prudents dans votre comportement, sinon le colis risque de vous diviser et de causer votre perte. » Mais ils n'y prêtèrent pas beaucoup attention. À un moment donné, ils eurent faim. Les deux aînés envoyèrent leur cadet acheter à manger et à boire, dans le village qu'ils venaient de traverser. Assis sous un manguier, les deux aînés songèrent avec cupidité au colis qui leur avait été donné (les dollars et le diamant); dans leur cupidité, ils ourdirent le projet funeste de frapper leur cadet dès son retour et de se partager à deux la somme d'argent.

Mais le cadet n'était pas non plus exempt de la même envie de s'approprier tout seul le contenu du colis. Aussi avait-il ourdi de son côté un projet funeste d'éliminer ses deux aînés, en mettant du poison dans le repas qu'il leur apportait.

Dès que le cadet apparut, les deux frères qui étaient cachés dans un buisson se ruèrent sur lui; le rouant de coups, ils le tuèrent. Cette bataille avait miné encore plus le peu de forces qui leur restaient, et aussitôt après avoir tué le cadet, ils se jetèrent sur la boisson et le repas qu'il avait apporté. Mais aussitôt qu'ils avaient mangé et bu, au moment de se partager le colis, des malaises mortels se déclarèrent chez l'un et l'autre, ils moururent sur place, laissant intact le colis des dollars et du diamant.

\subsection{Kabuluku le prétendant}

Un homme avait une fille unique, très belle. Lorsqu'elle atteignit l'âge du mariage, il en était tellement jaloux qu'il fixa une condition drastique pour quiconque voudrait l'épouser. La condition était pratiquement irréalisable: il fallait que le prétendant lui construisît une maison d'habitation en une seule journée. Les prétendants ne manquèrent pas de s'essayer. Le lion vint; il aimait et adorait la fille; mais il fut incapable de réaliser la condition posée. Il en fut de même pour plusieurs autres animaux.

Vint alors le malin Kabuluku (le renard), petit et mignon. On lui donna aussi l'occasion d'essayer. Il commença par alerter tous les membres de la famille des Renards, et partagea la tâche entre eux: un groupe s'occu- 
perait de couper les sticks dans la forêt pour les murs; un autre se chargerait du toit; un troisième s'occuperait des lianes et des nervures de bambou pour le haut et le bas de la structure; un quatrième groupe irait couper du chaume et viendrait couvrir la toiture; un dernier amènerait de l'eau et creuserait le trou pour ramasser la terre spéciale du mortier final qui couvrirait les murs en pisé.

Le jour fixé arriva, et chacun des groupes s'adonna à la tâche, au nom du seul Kabuluku prétendant. Dès 5 heures du matin, l'un après l'autre, les pairs Kabuluku, semblables en tout, apportèrent tous les matériaux nécessaires à la construction. Chaque groupe faisait son travail, et laissait la place à l'autre, le tout sous le commandement et la surveillance du seul Kabuluku prétendant. Vers 17 heures, le dernier groupe arriva; il creusa un trou profond, en sortit la terre rougeâtre et puisa de l'eau; le mortier fut pétri, et les murs en furent recouverts. À 18 heures, le prétendant en sueur se présenta chez le père de la fille et déclara terminés les travaux. Le père vint voir, et effectivement, la condition venait d'être remplie; c'est ainsi que par organisation et collaboration, en y mettant une grande finesse d'esprit, le petit Kabuluku épousa la plus belle fille de la région.

\section{Comment cette vie s'exprime-t-elle dans les fables?}

Généralement, les contes et fables, dont nous venons de voir un échantillon, interprètent la vie assez pénible que mènent les peuples de tout un continent, à partir d'une de leurs bases, depuis leur domination par les Occidentaux jusqu'à présent. Essayons de la saisir dans ses divers éléments. J'en mentionne quelques-uns, qui représentent l'ancien mêlé au nouveau.

Sont révélés sur le plan alimentaire: le repas habituel de base est composé de pâte de manioc mélangé au maïs (bidya), accompagné de viandes soit de volaille, soit d'animaux (chèvre, vache, gibier, poisson...). Les contes font état des détails intéressants de divers condiments: sauces, oignons, feuilles odoriférantes de forêt ou d'Europe, sels et poivres (voir par exemple le conte 1 ).

Les fables révèlent l'architecture locale: les maisons en chaume sont construites avec des sticks, des bambous de palmier, des lianes recueillies dans les forêts, du chaume coupé dans la savane, de la terre rouge pour faire le mortier qui recouvre les murs. Mais comme la vie actuelle comporte des éléments nouveaux, les fables qui sont contées actuellement les y ajoutent: ainsi y apparaissent des fenêtres, des portes en bois, des cadenas, des clés, des tôles, des matelas, des chaises, éléments tout à fait nouveaux, que le conteur ajoute pour suivre la trame de la vie (conte 8). 
Sur le plan technologique, les fables suivent également l'évolution de la vie. À certains endroits, le mortier ancestral cède la place au moulin électrique ou au moteur diesel; dans la conservation de la température des aliments, la calebasse ancienne cède la place aux thermos; $y$ apparaissent également des verres, des assiettes en porcelaine, récipients qui ne figurent pas originellement dans nos contes! L'automobile, les avions, les motocyclettes, les ordinateurs, les radios et télévisions sont empruntés par les personnages actuels de nos contes, qui ne dédaignent pas pour autant la pirogue et la houe.

Pour manifester encore mieux la manière dont nos contes et fables suivent la vie, rien de tel que l'apparition des métaux précieux et en particulier des diamants dans ces récits. Le rôle joué par le diamant au Congo dans la transformation sociale est énorme. On a vu, du jour au lendemain, un pauvre devenir riche, un village d'agriculteurs se transformer en un marché de grande envergure, grâce à l'apparition du diamant dans un de ses coins (conte 7). Des rituels sociaux nouveaux ont été élaborés dans ces milieux, telle la bénédiction des puits de diamants par le chef coutumier. Les nouveaux étalons d'échanges, tels la monnaie, les dollars, les titres fonciers, tout cela a fait irruption dans les fables anciennes, proclamées par de nouveaux conteurs.

Les fables racontées sont remodelées à propos des thèmes révélateurs de la vie des peuples dominés d'une périphérie du monde. Ce ne sont donc pas des récits d'antiquaires, destinés à la diversion, au détournement de la vie réelle faite de contradictions entre les sociétés africaines de la parenté (Augé 1975; Muena Muanza 1985) et la société dominante du capital (Marx 1969) qui vise la restructuration totale et totalitaire de la planète dans leurs options, imaginations et intérêts (contes 4 et 5). Fables et contes révèlent en résumé les piliers des sociétés en confrontation, la parenté, ses valeurs et pesanteurs et le capital, ses valeurs et avatars. Moins naïs qu'on ne les pense, les peuples d'Afrique, dans une de leurs sections de base, démontrent, à travers ces fables et contes, leur volonté et leur capacité à comprendre la société qui façonne le monde depuis près de 500 ans et qui utilise à son profit en Afrique la société de la parenté comme tuyau d'échappement et de sécurité. Les contes révèlent ainsi les deux vecteurs de base, l'argent et la parenté, autour desquels gravite la vie en Afrique, détruite de plus en plus à cause de leur mauvaise articulation. La parenté et sa valeur centrale de solidarité est souvent avalée par la recherche effrénée de l'argent (conte 7). 
Les quelques exemples que nous avons relatés au début de cette contribution stigmatisent en effet sans ambages, à la lumière de la société ancestrale d'harmonie et de solidarité, les fossés sociaux qui se creusent et s'accroissent de plus en plus depuis l'indépendance entre Africains riches et Africains pauvres (conte 1). Ces inégalités criantes sont malheureusement accrues et occultées par la parenté et principalement par sa solidarité mal comprise et mal gérée par les "pauvres ». La solidarité parentale apparaît comme une réelle pierre d'achoppement du capitalisme en Afrique noire. Elle est une carapace qui empêche la compréhension des inégalités sociales et qui tempère et annule la lutte des classes en la gommant sous la lutte factice des clans, des ethnies, des régions (conte 6).

Les contes et fables actuels d'Afrique noire stigmatisent aussi les rapports de force entre l'«Euramérique » et l'Afrique ${ }^{6}$, le pouvoir arbitraire et la mainmise dictatoriale de l'Europe sur l'Afrique, ses structures et leurs animateurs dans les domaines différents de la politique, de l'économie, du phénomène religieux et de l'éducation. Apparaît aussi comme en filigrane, dans ces contes, que cette mainmise occidentale est réussie grâce à la transformation totale (corps et esprit) des fils et filles d'Afrique, qui n'osent pas affronter courageusement la société capitaliste, malgré l'indépendance politique, en la restructurant fondamentalement et en la refondant sur une autre vision et sur une autre logique (conte 5).

$\mathrm{Au}$ lieu, par exemple, de créer un système économique nouveau du «serrons les coudes au travail, pour que nous puissions partager à plusieurs nos profits ", par rapport à l'ancien qui prônait que plusieurs se partagent ce qu'un seul a produit ${ }^{7}$, et par rapport au système capitaliste de la production sociale maximale pour une répartition égoïste ( «serrez les coudes au travail, pour augmenter le profit de l'entreprise $\left.{ }^{8} »\right)$, les contes actuels montrent comment les Africains se complaisent dans l'envie, dans la poursuite chimérique de la richesse, dans l'utilisation des fétiches, dans le commerce sexuel, dans la mendicité, dans les solutions illusoires: entraide avec les parents; exil politique et économique; fuite de cerveaux, etc.

6. Beaucoup d'auteurs critiquent l'impérialisme occidental, entre autres, Ziegler (1980; $1988 ; 1998 ; 2002 ; 2005)$.

7. Selon le proverbe Cyadime umwe, cyàdya bângi (plusieurs se partageront ce qu'a produit un seul).

8. Ce qui donne en langue Luba: cyadime bangi, cyadya bamwe. 
Les contes racontent à profusion l'éclosion en Afrique d'un capitalisme sauvage dans lequel les Africains pataugent dans tous les secteurs de la vie, sans penser à évoluer vers un capitalisme industriel. Ils relatent la vie d'un continent bloqué dans le capitalisme commercial et dans les méthodes d'accumulation primitive du capital, décrites par Karl Marx, méthodes immorales de pillage, de tuerie (luttes fratricides), de tricherie, de corruption, de vente et d'achat de tout (dont le pouvoir), du savoir, du sacré (conte 6).

Ce qui se passe chaque dimanche, dans une célébration où un conte est proclamé par quelqu'un de la communauté, est une grande révolution, une véritable bombe dans la vie politique du pays. Ainsi, par exemple, certains contes mettent clairement le doigt sur le manque de vision politique révolutionnaire dont l'Afrique a besoin. Ils décrivent la vie politique africaine faite d'intrigues incessantes, le pouvoir arbitraire, la dictature des dirigeants, leur utilisation abusive des biens collectifs, leur enrichissement illicite et leur investissement dans la construction des villas, dans le mariage polygamique ou dans la prostitution, l'attachement au pouvoir comme instance d'enrichissement, la clochardisation du pouvoir coutumier, un système juridique injuste, une justice prisonnière du pouvoir, un pouvoir à la Machiavel, les rapports de force en faveur d'une clique au pouvoir et au détriment du peuple, etc. Pour cette clique au pouvoir, toute autorité, en famille, en économie, en politique, en religion ou dans les écoles est considérée comme pouvoir dictatorial du Prince, acquis par tous les moyens, au profit de l'intérêt personnel. Les pays divers du monde noir, dans maints domaines, n'apparaissent-ils pas comme des jungles, des lieux où l'homme est un loup pour un autre (bomo homini lupus), où les gros poissons mangent les petits, où s'ourdissent des projets fratricides?

Nombreuses reprises de contes à ce propos suggèrent des idées et des inspirations dont manquent cruellement nos sociétés actuelles d'Afrique noire. Durant près de 50 ans d'indépendance formelle, nous n'avons jamais confronté courageusement les structures politiques héritées de la colonisation, ni la logique du pouvoir souverain du Prince en vigueur, avec la logique africaine du Chef, fondée sur la vérité, la solidarité et le service (Kabongo Kanundowi et Bilolo Mubabinge 1994).

Les rapports de force instaurés contre l'Afrique dans le domaine des religions ne sont pas moins relatés. Sur ce plan des convictions religieuses des peuples, la guerre des religions est déclarée. Il n'y a pas de rencontre dialectique entre les religions ou les cultures importées et les religions ou cultures autochtones. Les religions africaines sont traitées de « rétrogrades, 
inférieures ", alors que la révolution religieuse exige le perfectionnement des religions locales au contact de celles importées. Cinquante et un ans d'indépendance du continent n'ont jamais produit des changements majeurs des structures religieuses importées au profit de l'Afrique. Le recours aux «instances invisibles» alliées de la vie (contes 2 et 7 ) incite à concevoir un type de religions nouvelles de libération de la vie africaine, menacée individuellement et collectivement; il incite à quitter des religions devenues des «portes de sortie » vers l'ailleurs, des lieux d'implosion de la cupidité, de la concupiscence, de la pédophilie, du tribalisme.

Les contes et fables d'aujourd'hui relatent en fait et en résumé la vie d'une Afrique mal partie, depuis son indépendance factice, incapable de mener sa propre révolution, au lieu de n'être qu'une caisse de résonance, un berceau d'imitations serviles et caricaturales (conte 3). Mais il y a plus dans ces contes et fables: il y a indication d'une option de la résistance à la sujétion, de la solution difficile d'affronter sa propre situation misérable afin de la transformer en la refondant sur les valeurs sûres du travail collectif, de la redistribution équitable, de respect de la vie, de toute vie.

Ce sont ces différents thèmes qu'explorent les divers contes déclamés dans nos assemblées dominicales de Cikapa-Kele. Je n'ai rien inventé. Le conte 1 traite entre autres de la boisson comme d'une bombe larguée stratégiquement par quelqu'un ou un peuple, dans la scène sociale. La boisson enivre et fait perdre conscience, exposant la vie à la mort. Elle est donc une grande faiblesse pour tout homme. Les peuples autochtones d'Amérique du Nord ont péri entre autres à cause de la boisson alcoolisée. La fable montre aussi les forces sociales qui vivent aux dépens des autres comme des sangsues, réalisant leurs rêves (aspirations) au-dessus de leurs moyens. Les pauvres font des cérémonies festives coûteuses (mariages, deuils à Kinshasa, sorties somptueuses de maternité, collations tumultueuses de grades académiques, fêtes du mouton chez les musulmans, etc.) avec les biens des autres, soit par solidarité, soit par emprunts... Le récit aborde aussi en même temps le problème des dettes éternelles fréquentes, qu'on ne veut pas payer en Afrique. Plusieurs foyers vivent de dettes. Il en est ainsi des Églises, des pays... Ils sont endettés jusqu'au cou. Est donc stigmatisée toute aspiration de vivre matériellement bien, par des moyens malhonnêtes (détournement des fonds d'autrui, non-transparence de gestion, débauches, etc.). Et enfin, on y indique le règne de la jungle qui caractérise le monde actuel. Le développement des uns repose sur le sous-développement des autres (voir Amin 1973, 1976, 1978 ; Frank 1971, 1972; Wallerstein 1966, 1980). 
Le récit 2 parle de celui qui tend les pièges (stratège), piégé lui-même par les femmes qui lui exposent leurs seins et ventres tatoués. Le corps humain (féminin surtout) nu est une bombe stratégique de détournement ou de léthargie. Le récit traite aussi du recours aux instances invisibles alliées de la vie. Cette alliance avec le monde invisible aguerrit un peuple. Elle permet de juguler la faim et la mort des membres de famille. L'instance invisible alliée de la vie apprend les stratégies de sortie de piège (crises): la tentative d'oser toucher les seins des femmes d'autrui (geste coupable). Voilà le geste qui, sans aboutir, sauvera la situation.

Le récit 3 stigmatise l'attitude qui consiste à envier les autres. Chacun doit vivre de ses talents, de ses ressources naturelles et culturelles, en profitant de celles de l'autre et non en l'enviant. Chacun devra répondre de ses dons, de ce qu'il en aura fait, devant Dieu et devant l'humanité. Or, l'Afrique actuelle meurt d'envie et d'imitations caricaturales; car "celui qui ne fait qu'envier ce qu'ont les autres, finit par perdre ce qu'il a en propre » dit un proverbe luba.

Le récit 4 désigne métaphoriquement les rapports de force et la dictature qui existent entre les hommes, entre les continents et entre leurs structures sociales. Quand de tels rapports d'exploitation et d'instrumentalisation des hommes entre eux sont vécus aux niveaux familial, scolaire, administratif, politique, économique, religieux, etc., cela provoque une destruction générale de la société. Le pangolin symbolise métaphoriquement les forces sociales marginalisées et exclues de la gestion de la vie sociale, et qui doivent s'insurger et donner de la voix.

Les récits 5 et 6 détaillent les magouilles et combines de pouvoir qui empoisonnent nos régions, depuis que le pouvoir coutumier traditionnel a été confisqué par les gouvernements modernes, et mis à genoux. Ceux qui étaient naturellement investis comme chefs, doivent aujourd'hui ramper devant des individus auxquels le peuple n'a donné aucun pouvoir, et qui prétendent gérer nos peuples.

Le récit 7 explore le vecteur argent comme source de beaucoup de déboires sociaux. L'argent guide aveuglément le monde «moderne». Tous les moyens sont bons pour l'avoir. Il déclenche des projets guerriers qui engendrent l'esclavage, la colonisation, le pillage et cause des millions de morts. Il engendre en Afrique des guerres fratricides, des génocides. Le récit montre aussi la fascination que les Africains ont de la richesse et non des moyens honnêtes de s'en procurer tels que le travail social intense, par exemple. Il montre en outre comment, plus altruistes dans leur culture, les Africains deviennent de plus en plus égoïstes dans leur vie. Ils veulent 
s'accaparer seuls la richesse, à l'exclusion des autres. Nous sommes loin du rêve du socialisme africain.

L'on voit ainsi les Noirs utiliser les biens publics - l'État, l'Église dans leur intérêt égoïste comme des dépotoirs, des poubelles. C'est le pillage et la privatisation outranciers des institutions étatiques, des caisses diocésaines, des paroisses... D'où la lutte pour le maintien des uns et des autres à des postes de direction, pour l'éternité. Le récit explore encore davantage le vecteur de la richesse, moteur de la société capitaliste d'après Smith (1976), Ricardo et Marx. La cupidité et l'égoïsme de consommation rapide y sont dénoncés. Qui, aujourd'hui, peut exhiber sa richesse en toute transparence devant sa femme, son mari, tout le village, comme une richesse acquise pour tous?

Le récit 8 parle d'un mariage assorti d'une condition difficile de réalisation: la construction d'une maison en un jour. La stratégie d'union et de collaboration est indiquée comme centrale pour arriver à réussir un projet juste. L'union, et non la division, qui reste la devise pour dominer (divide et impera).

Mais la vie n'apparaît pas seulement dans la manière dont la société est décrite dans ses moindres structures d'organisation et ses rapports de force. Elle est exprimée aussi dans la manière dont les contes et fables d'Afrique noire sont déclamés, une manière vivante. Les fables ne sont pas destinées à être lues en Afrique noire, mais à être dites, par un être vivant; elles sont déclamées, avec les contours de la vie de celui qui raconte. Aussi la fable prend-elle la coloration de la vie de celui qui la raconte. Et c'est ainsi qu'elle est inépuisable, car elle semble toujours neuve, tant qu'elle est racontée par différentes personnes. Dans son livre sur la catéchèse de l'oralité en Afrique, le professeur Kabasele Lumbala (1997) montre un exemple de la fabrication des fables à partir des données déterminées d'un récit précédent. L'auteur tire cet exemple bien connu de la parabole du semeur, où un même don fructifie différemment selon la disposition des personnes qui le reçoivent: les grains tombés sur le chemin, les grains tombés parmi les ronces et épines, les grains tombés sur un sol rocailleux, les grains tombés sur une bonne terre fertile et préparée (Mt 13,1-9). Un même récit peut être raconté par une dizaine de personnes, chacune selon les harmoniques propres de sa vie, de son milieu, de ses expériences antérieures. Dans une catéchèse qui met à profit ces harmoniques du conte africain, l'auteur invite non seulement à étudier les contes, mais surtout à les recréer à partir des éléments que nous en connaissons. Jésus, qui était de tradition orale, a pu créer un récit, brodé sur l'observation de la vie quotidienne et de la 
nature. À Cikapa-Kele, nous essayons de faire de même, en brodant le même message sur des éléments du milieu de notre travail, du milieu de notre groupe...

Voici un exemple tiré du livre que nous venons de citer. Nous sommes dans un centre de soins médicaux. On lit le texte Mt 13,1-9. Puis quelqu'un raconte:

Le médecin arrive pour soigner les malades; il en trouve de plusieurs catégories: ceux qui toussent, ceux qui boitent, ceux qui sont ambulants, ceux qui sont grabataires, ceux qui ont la fièvre, ceux qui transpirent, ceux qui sont maigres, ceux qui sont obèses... Il les salue tous, leur serre la main; il pose des questions, note sur son papier, ausculte, regarde dans les yeux, sur la langue, etc. Puis il donne des médicaments à tous. Mais les malades ne sont pas toujours en harmonie avec les médicaments qu'ils reçoivent.

Il y a des malades trop minés par des vers intestinaux; ils n'arrivent pas à digérer ce qu'ils avalent, et tout s'écoule au dehors aussitôt après. Parfois même les vitamines qu'on leur administre, à peine avalées, sont-elles ingurgitées par des vers auxquels ces vitamines profitent plus qu'aux corps euxmêmes.

Il y a des malades qui sont à l'extrémité de leurs forces physiques et qui n'ont même plus la force d'avaler les médicaments. On arrive à les leur faire prendre à grands efforts; mais à peine le médicament parvenu à l'estomac, qu'il ressort par des vomissements. Il y a des malades capricieux avec les médicaments; parmi eux un certain nombre ne croient pas en l'efficacité de la thérapie entreprise, soit parce qu'ils n'ont pas confiance en eux-mêmes, soit parce qu'ils sont hostiles au médecin. Ceux-là, dès qu'on leur laisse du médicament, ils font semblant de l'accepter, mais aussitôt les infirmiers partis, ils le jettent ou l'oublient au fond du tiroir; c'est ainsi qu'on trouve des tiroirs de malades pleins de comprimés.

Il y a aussi des malades qui arrivent à prendre le médicament, mais n'observent pas les conditions requises, par exemple l'abstention des boissons alcoolisées, des viandes fumées, du sel ou du sucre, etc. Ces exigences n'étant pas observées, le médicament qu'ils prennent n'arrive pas à produire ses effets.

Il y a enfin des malades qui acceptent le médicament de bon cœur, conscients de leur maladie, et qui en le prenant, guérissent, car ils observent les exigences du médecin ainsi que les dispositions de la prise du médicament, et guérissent. (Kabasele Lumbala 1997) 


\section{Conclusion}

L'homme créé à l'image de Dieu doit continuer à créer, à progresser en gagnant d'autres talents avec ceux que Dieu lui avait remis. Et toute communauté humaine est investie du même appel et pouvoir. Ces paroles que j'ai écrites il y a une quinzaine d'années, résonnent toujours comme actuelles:

Mission historique et inédite pour chacune des communautés chrétiennes, et aucune ne doit parler et agir par procuration. Tout ce qui est imaginé, senti, pensé et essayé sur le sol africain de Budikadidi, Saint Joseph, pour être saisi à sa juste valeur, doit être compris dans ce sens de la liberté et de la créativité des Africains actuels qui s'y trouvent dans toute leur densité historique. Il n'est pas question sur cette portion de terre de simplement réitérer les expériences millénaires, peut-être vénérables des Églises européennes. Il n'est pas question non plus de simplement répéter les expériences séculaires de nos «aïeux» [...] C'est une sorte de recréation, de renouvellement fondamental des christianismes constitués, précédents, sur toutes les facettes de leurs productions. C'est l'exercice des fonctions imprescriptibles d'une communauté africaine de chrétiens dans les domaines de la prière, de la morale, du droit, de la pensée théologique et de leurs implications matérielles. (Muena Muanza 1995, 38-39)

Ce sont ces efforts qui nous ont fait découvrir la vitalité des contes et fables de nos traditions africaines: ils sont pleins de vie, et constituent des ressources inépuisables de matériaux d'enseignement et d'entraînement social; car il y a la vie qui grouille dans les fables et contes, et qui interpelle constamment et continuellement la vie que mènent les hommes et femmes d'aujourd'hui.

\section{Références}

Amin, S. (1973), Le développement inégal, Paris, Minuit. (1976), L'impérialisme et le développement inégal, Paris, Minuit. (1978), L'accumulation dépendante, Paris, Anthropos.

(1988), Impérialisme et sous-développement en Afrique, Paris, Anthropos.

Association culturelle Sangalayi (1996), Les tambours des guêpes, conte luba, Bruxelles, Éditions Sangalayi.

Augé, M. (1975), dir., Les domaines de la parenté, Paris, Maspero. 
Frank, G. (1971), Lumpenbourgeoisie et lumpendéveloppement, Paris, Maspero.

(1972), Le développement du sous-développement, Paris, Maspero.

Kabasele Lumbala, F. (1997²), Catéchiser en Afrique aujourd'hui, apport des traditions orales, Kinshasa, Baobab.

Kabasele Lumbala, F., et al. (2011), Naissances insolites en terre africaine, Paris, Karthala.

Kabongo Kanundowi, E. et Mubabinge, B. (1994), Conception bantu de l'autorité. Baluba; bumfumu ne bulongolodi, Munich, Publications universitaires africaines.

Marx, K. (1969) [1867], Le capital. Livre I, Paris, Garnier-Flammarion.

Muena Muanza, N. (1985), Chants et métaphores populaires du Kasayi: pour une relecture du vécu social au Zaïre, de 1885 à 1982, Thèse doctorale, Québec, Université Laval.

(1995), "L'expérience de Cikapa-Kele, essai d'une communauté chrétienne africaine de se prendre totalement en charge ", Revue africaine des sciences de la mission, 3 .

Muena Muanza, N. et Kabasele Lumbala, F. (2001), Kutendeleela Yezu mu bwena kwetu (Célébrer Jésus dans nos cultures), Donzdorf, Éd. Bafiike dimanyayi.

Sмiтh, A. (1976), Recherches sur la nature et les causes de la richesse des nations, Paris, Gallimard.

Wallerstein, I. (1966), L'Afrique et l'indépendance, Paris, Présence Africaine.

(1980), Le Système du monde du XVe siècle à nos jours. Vol. 1 Capitalisme et économie-monde, 1450-1640, Paris, Flammarion.

Ziegler, J. (1980), Retournez les fusils, Manuel de sociologie d'opposition, Paris, Seuil.

(1988), La victoire des vaincus, oppression et résistance culturelle, Paris, Seuil.

(1998), Les seigneurs du crime. Les nouvelles mafias contre la démocratie, Paris, Seuil.

(2002), Les nouveaux maitres du monde et ceux qui leur résistent, Paris, Fayard.

(2005), L'empire de la honte, Paris, Fayard. 


\section{Résumé}

CEuvre de liturgie et d'anthropologie esthétique, cette publication révèle la vitalité d'une communauté chrétienne d'Afrique centrale (du Congo Kinshasa, à Cikapa) qui affronte ses problèmes de survie, dans une liturgie eucharistique dominicale, émaillée de contes comme quatrième lecture. Ces contes y apparaissent comme un moment important de Révélation en Afrique et un moyen mnémotechnique puissant d'éducation et d'action populaire. Y est interprétée, de manière critique et métaphorique, la vie des sociétés postcoloniales, dominées par un capitalisme commercial sauvage avec ses méthodes immorales d'accumulation, au détriment de la majorité et au service d'une minorité interne liée à l'impérialisme. La vie apparaît aussi dans la créativité artistique même des contes qui ne sont jamais figés et dans la manière vivante de les déclamer. Les contes sont une constante réinterprétation de la vie dans le miroir culturel africain et chrétien.

\section{Abstract}

This paper, which draws from both liturgical and anthropological studies shows the artistic creativity that can be at work in black African Christian communities, more specifically the community of Tshikapa, in West Kasayi (DR Congo). This Christian community uses African traditions as a fourth biblic reading passage in the liturgy, in order to show how they can courageously address the problem of their survival. Through oral tradition, they try to describe and interpret their difficult, painful African post-colonial life, thus reinventing their culture and church life. 